\title{
PERFORMANCE TERMO-ENERGÉTICA ENTRE DOS TIPOLO- GÍAS DE ENVOLVENTES DE CONSERVACIÓN DEL VINO
}

\author{
-Dra-Arq. Ramos Sanz, Alba I. \\ Consejo Nacional de Investigaciones Cientificas y Tecnológicas (CONICET) - Facultad de Arquitectura, \\ Urbanismo y Diseño (FAUD) de la Universidad Nacional de San Juan (UNSJ) - Instituto Regional de \\ Planeamiento y Hábitat (IRPHa) - Av. Ignacio de La Roza y Meglioli - 5400 San Juan - Argentina \\ Tel.:+54(0)264 4232395 / 3259 - Fax: +54(0)264 4235397 - http://www.irpha.com.ar - \\ e-mail: aramossanz@faud.unsj.edu.ar; draarqalbaramossanz@gmail.com
}

Palabras clave: eficiencia, demanda termo-energética, simulación, vinificación .

\section{Resumen:}

Se aborda el problema energético de la agroindustria representativa de Cuyo. El desempeño térmico de las envolventes afecta a los ámbitos de producción de alimentos y bebidas, dado que este factor condiciona la demanda energética de la agroindustria. En la elaboración de vino existen unidades de fermentación tecno lógicamente diferentes que determinan una demanda térmica en función de su interacción con el clima cálido de San Juan. En este trabajo se analizan los tipos tecnológicos productivos en función de la interacción clima-envolvente, a partir de los datos cuantitativos de modelización, simulaciones térmicas y seguimiento con sensores. Se relevan las envolventes de conservación del vino de dos materiales tradicionales de la zona: adobe y ladrillo encadenados. A fin de registrar el comportamiento térmico de las envolventes de conservación del vino, se lleva a cabo un seguimiento con sensores de temperatura interior, exterior e irradiancia solar durante un periodo cálido. Con los datos recogidos in situ y los datos estadisticos del clima árido, se modelan los casos para la simulación térmica. Los resultados indican razones para definir la conveniencia termo-energética entre ambos tipos de envolventes de conservación del vino.

\section{Introducción:}

Las estadisticas señalan que el $68 \%$ del volumen de vino se fermenta en vasijas de vinificación de mampostería y hormigón armado, a las cuales llamaremos en este estudio vasijas tradicionales (INV; 2008). Este factor tiene su origen en los elevados costos de importación, instalación y mantenimiento (Udaquiola et al, 2006; Merino, 2007) de los tanques de acero inoxidable o vasijas modernas. Asimismo la situación económica local reduce la rentabilidad de los productos vínicos obtenidos en vasijas de acero inoxidable (Min. de Producción de la Provincia de San Juan, 2010). Estos factores promueven la fermentación en tradicionales cascos de grandes volúmenes y sistemas geotérmicos de refrigeración (Min. de Relaciones Exteriores, 2012). Este estudio aborda el caso de las envolventes de conservación del vino, el cual no dista bastante de las envolventes de fermentación. Si bien se recurre también en este caso al empleo de tanques de acero inoxidable para la conservación del vino, aun es práctica frecuente utilizar depósitos o cisternas de materiales tradicionales. Se asocia este hecho a algunos factores que se mencionan a continuación: las prestaciones térmicas de dichas envolventes -evitando la dependencia de sistemas de control térmico-, la diversidad de volúmenes disponibles y la optimización del uso del espacio de la bodega de conservación.

Pero, así como en la actualidad los tanques de acero inoxidable desplazan a los tanques de acero negro y piletas de hormigón armado y ladrillo encadenado, en un principio - antes de la globalización del vino- las 
piletas y cisternas de ladrillo desplazaron a las cisternas de adobe encadenado. Este hecho, sucedido a fines del S.XIX y principios del S.XX, determina el desuso de un gran número de establecimientos industriales considerados tecnológicamente obsoletos. En la presente investigación se indaga sobre el potencial termo-energético actual de los antiguos cascos de conservación del vino mediante dos casos testigos, en comparación con las piletas de ladrillo y estructura de hormigón armado.

\section{Descripción del Área de Estudio:}

\section{Temperatura y Vientos:}

El Instituto Argentino de Normalización y Certificación (IRAM 11603; 1996) ubica el Valle del Tulum dentro de la zona Bioambiental IIla, con amplitudes térmicas diarias superiores a los +/- $14 \mathrm{~K}$. Los vientos en San Juan son de dirección predominantemente Sur a lo largo del año. La velocidad de los mismos se duplica en verano, alcanzando una media de $13 \mathrm{Km} / \mathrm{h}$.

\section{Intensidad de la radiación solar:}

Los sensores HOBBO U12 T12 registran hasta 850

$\mathrm{W} / \mathrm{m}^{2}$ en horas del mediodía en verano. Estos valores son semejantes a los aportados por el Handbook of Fundamentals de ASHRAE، Cap. VII, Tabla 15, para $40^{\circ}$ de Latitud (en Transferencia de Calor y de Masa, 2007). En verano, los aportes solares son significativos sobre el plano horizontal y aquellos planos orientados este-oeste. En invierno, la superficie de la envolvente más cálida se orienta al norte.

\section{Metodología:}

Se lleva a cabo un relevamiento de dos casos comparables. Se miden dos tipologias de adobe y de ladrillo con estructura de hormigón armado. Ambos casos se ubican en un mismo establecimiento. Actualmente se emplean los casos para la conservación del vino. Con el empleo de sensores, se recogen datos empíricos de temperatura exterior $\left({ }^{\circ} \mathrm{C}\right)$, irradiancia solar $\left(\mathrm{Wh} / \mathrm{m}^{2}\right)$, temperatura interior ( ${ }^{\circ} \mathrm{C}$ ) y humedad relativa (\%). Los equipos de medición HOBBO U12 T12 proveen 2459 datos para cada caso, y para el ambiente exterior (a la sombra). Las mediciones se realizan a cada minuto, durante cincuenta dias corridos. Los datos medidos se procesan en planillas de cálculo PROMEDI HTL (Blasco Lucas et al, 2007). Con estos datos empiricos se definen valores medios a partir de los cuales se modelan las envolventes de los casos en régimen estacionario y cíclico estacionario. La simulación térmica comprende variables independientes tales como la intensidad de la radiación solar, la velocidad de los vientos, el flujo de convección natural en el interior y exterior de la bodega, estimadas semi-empiricamente.

\section{Casos de Estudio:}

Se seleccionan dos casos de estudio, ubicados en el mismo establecimiento, por disponer de configuraciones geométricas y relaciones situacionales similares. Se pueden caracterizar los casos de análisis según;

-Capacidad: el caso 3 o EV adobe tiene $24000 \mathrm{~L}$ de capacidad, casi la mitad del caso 6 o EV ladrillo.

-Materialidad: el caso 3 dispone de muros de adobe con estructura encadenada, y el caso 6 dispone de mampuestos de ladrillo, con estructura reforzada.

Ubicación: ambos casos se ubican en la misma bodega, son unidades aglomeradas a otras, sobre rasantes.

\section{Umbrales Empíricos:}

Se sintetizan las temperaturas de conservación registradas en las diferentes EV monitoreadas. Estos constituyen los valores medios tanto para la conservación de vinos comunes como varietales. Se contemplan 18400 datos empíricos promediados con los valores teóricos medios, definidos como recomendada promedio. La temperatura media de diseño (TM) para verano es 22,2 ${ }^{\circ} \mathrm{C}$ y para el invierno es $15^{\circ} \mathrm{C}$. La desviación estándar en ambos casos es de $+/-1,9 \mathrm{~K}$.

El Clima en las zonas de confort:

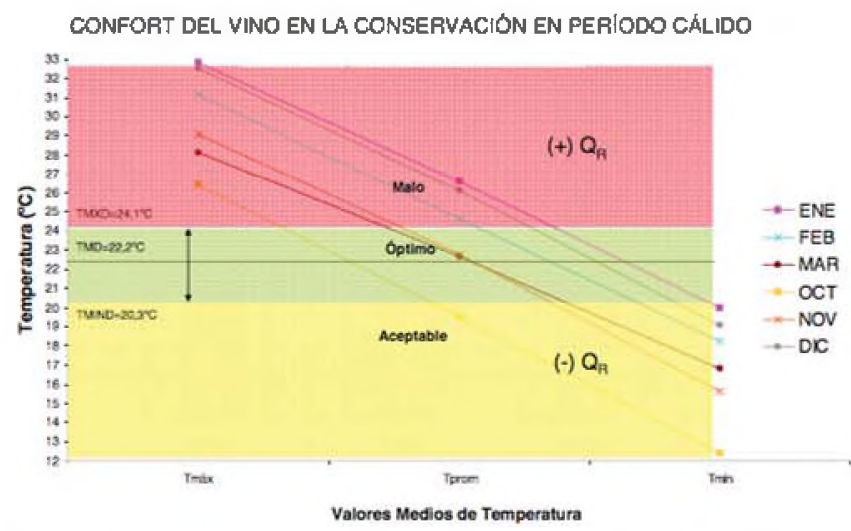

Figura 1: Delımitacion de las condicumes higrotérmicas adecuadas pare winos comunes y varietales para periodos caildos. 


\begin{tabular}{|c|c|c|c|c|c|c|c|c|c|c|c|c|}
\hline \multirow{3}{*}{ CONFORT } & \multicolumn{2}{|c|}{ ENE } & \multicolumn{2}{|c|}{ FEB } & \multicolumn{2}{|c|}{ MAAH } & \multicolumn{2}{|c|}{ क्सा } & \multicolumn{2}{|c|}{ ROV } & \multicolumn{2}{|c|}{ पाद } \\
\hline & & & & & & AIOD & CALII & & & & & \\
\hline & $\%$ & QA & $\%$ & QA & $\%$ & QR & $\%$ & QR & $\%$ & QA & $\%$ & $\overline{\mathrm{QR}}$ \\
\hline Malo & 68 & $(+)$ & 50 & $(+)$ & 40 & $(+)$ & 15 & $(+)$ & 35 & $(+)$ & 60 & $(+)$ \\
\hline Aceptable & 7 & $(t)$ & 15 & $(+)$ & 30 & $(+)$ & 60 & $(+)$ & 40 & $(+)$ & 15 & $(+)$ \\
\hline Optimo & 25 & $(-)$ & 35 & $\frac{1}{(-)}$ & 30 & $\frac{1}{(-)}$ & 25 & $\frac{1}{(-)}$ & 25 & $\theta$ & 25 & $\frac{1}{(-)}$ \\
\hline
\end{tabular}

Tábla 1:Porcentaje de rangos térmicos de los mases de conservacián an periodo cálıdo que inciden sobre el confort del wno.

Se emplea un gráfico de temperaturas sobre el cual se trazan los valores máximos, minimos y medios de cada mes. La extensión total de la línea del mes comprende el $100 \%$ de los datos climáticos promediados. Como indicador gráfico de confort se recurre a la estimación porcentual de ingreso en la zona de confort de la línea del mes. Cuando dicha línea supera la zona de confort hacia arriba, indica una sobrecarga del sistema de refrigeración, definido como (+) QR. Cuando la línea supera la zona de confort hacia abajo, se designa como (-) QR y representa una reducción de la demanda de frío o de la DE. Las zonas de confort del vino y las líneas de temperatura de cada mes son valores medios, afectados indirectamente por las propiedades de la EV, pero $\sin$ ser especificadas.

Se observa que los meses de Enero (rosa), Febrero (cian) y Diciembre (gris) presentan más de la mitad de los valores térmicos sobrepasando las temperaturas máximas de confort del vino en conservación. En Enero se debe accionar un sistema de control térmico durante la jornada completa, ya que las temperaturas mínimas dentro del área de confort, no inducen un refrescamiento del vino. La situación es similar en Diciembre.

En Octubre la conservación se puede llevar a cabo dentro de una EV de gran resistencia térmica, sin necesidad de recurrir a sistemas de control térmico. Presenta los menores porcentajes de sobrecarga térmica entre los meses de período cálido.

\section{Monitoreo Térmico}

El monitoreo demuestra dos vasijas con un comportamiento relativamente estable térmicamente.

Sin embargo, al superponer la zona térmica apropiada a la conservación del vino, se observa que la vasija de ladrillo supera la misma, mientras que el caso de la vasija de adobe se encuentra dentro de la zona, a lo largo del período considerado. También se observa que la temperatura en la EV de adobe se ubica por encima de la media $\left(22,2^{\circ} \mathrm{C}\right)$ durante todo el período.
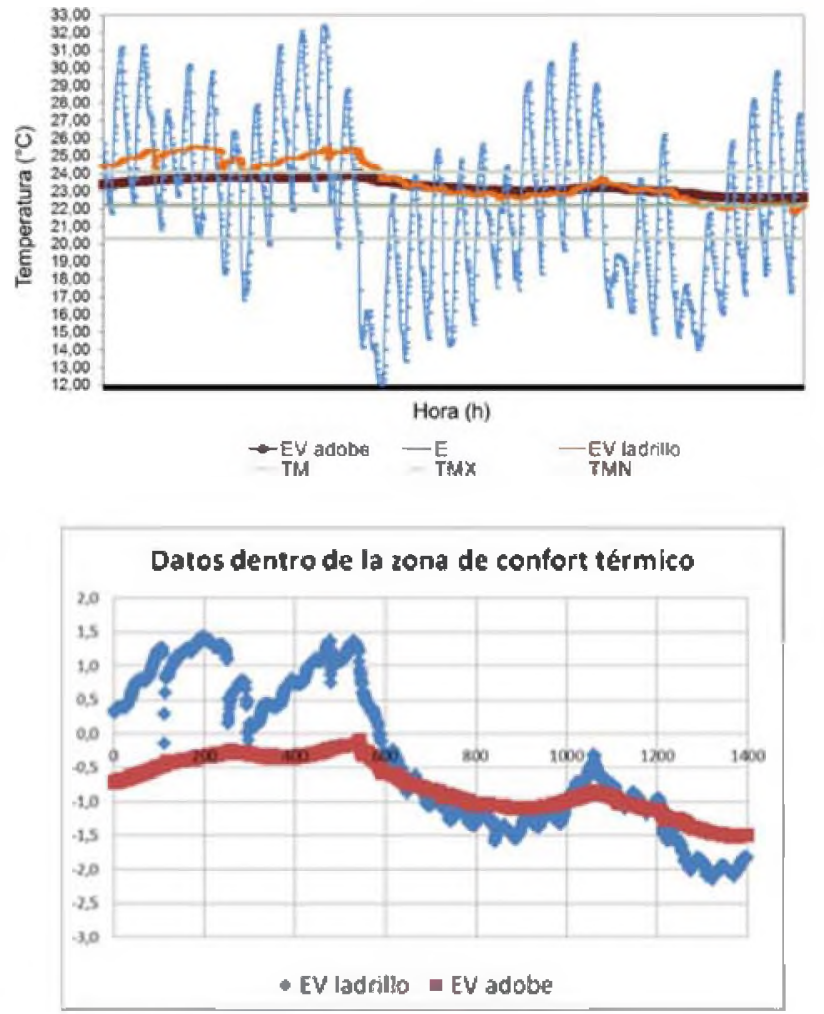

Figura 2: Segumtiento térrinco de los casos analizados, en la proyerctón de la zosa térmica de conservación del vina.

Figura 3: Población y dispersión de los datos en relación a la temperatura maxima de conservación dell vino.

Se observa que la performance térmica de la vasija de ladrillo (EV ladrillo) escapa a la zona de confort de conservación del vino en período cálido. De un total de 1400 datos, 600 datos de la EV de ladrillo superan el rango máximo de temperatura para la conservación del vino. Esto constituye un $42,85 \%$ del tiempo, la masa de vino se "calienta" por demás. El tiempo relativo que el caso trasciende las condiciones térmicas adecuadas coincide con los porcentajes estimados para el mes de Marzo con un $60 \%$ del rango dentro del área de confort. Esto se explica porque el método gráfico utiliza los valores medios de temperatura, y las envolventes tradicionales proporcionan un amortiguamiento suficiente para mantener el vino a la temperatura media de un período medianamente prolongado. Si se tratara de envolventes no tradicionales, el vino trascendería los valores medios del aire exterior, dado el reducido amortiguamiento de las mismas.

Los motivos de esta diferencia entre ambas envolventes podrían develarse a partir de la experimentación sobre diversas variables, por medio del modelado térmico semi-empírico.

\section{Modelo Fisico}

Para obtener el modelo térmico, se plantean las siguientes suposiciones de partida; 
a. La masa de vino se considera homogénea en todo su volumen e isotérmica.

b. La transferencia de calor es unidireccional y perpendicular al plano de la superficie de los muros, cubierta y piso-envolvente- de los fermentadores.

c. El límite del modelo comprende la radiación solar diurna sobre el plano de la vasija y la emisión de larga longitud de onda hacia el cielo, en ausencia del sol.

d. El límite del modelo aborda la convección térmica interior y exterior.

e. La conducción de calor a través de los elementos de la vasija y del mosto se estima en régimen estacionario y cíclico estacionario, considerando el efecto de la inercia y retardo sobre la temperatura del vino.

f. La transferencia de calor entre vasijas tradicionales adosadas o apiladas se considera igual a cero, dado que no hay diferencia de temperatura entre ellas al destinarse ambas a la fermentación.

g. Los nodos o células del modelo se ubican en el núcleo del mosto, contenido en el centro volumétrico gravitacional de la vasija.

h. La vasija se estudia como un modelo monozona.

El coeficiente combinado hi es función de los intercambios superficiales por radiación y por convección, simultáneamente. La estimación de la convección natural o forzada (hic), se propone por Mc Adams (1954, en Colombié et al) quien proporciona algunos modelos empíricos para obtener $h_{\text {r.; }}$;

$$
\mathrm{h}_{\mathrm{t}}=\frac{\mathrm{Nu}^{\star} \lambda_{\text {Nhido }}}{\mathrm{H}}\left[\mathrm{W} / \mathrm{m}^{\mathrm{20}} \mathrm{C}\right](1)
$$

\section{Donde:}

- Nu es el número adimensional de Nusselt o módulo adimensional de transferencia de calor por convección. - Anido es la conductividad térmica, en este caso del agua que es igual a $0,6 \mathrm{~W} / \mathrm{m}^{\circ} \mathrm{C}$.

- H es la altura del tanque.

El intercambio convectivo alrededor de una EV puede darse por medio de convección natural o forzada. En la primera, el flujo de calor es vertical incrementándose con la altura y con la diferencia de densidad. La convección forzada se observa en EV expuestos a la intemperie o en salas ventiladas (Colombié, S. et al, 2006), en los cuales el aire fluye perpendicularmente alrededor, a temperatura constante. Este es el caso representativo y se expresa como;
$\mathrm{Nu}=0,32+0,43^{\star} R e^{0.52} /-1(2)$

Para $h_{1} h_{c}$ es función de las propiedades termodinámicas del vino. Normalmente se

supone que $R_{s}>R_{s e}$

\section{Resultados y Discusiones:}

Existen algunas variables comparables en ambas tipologías de envolventes productivas:

a) Las propiedades térmicas de la envolvente ( $R, K)$

Se observa que las transmitancias térmicas son mayores en el caso de la EV de ladrillo. Tanto en horas del día como de la noche, el desempeño térmico de la envolvente de adobe es favorable a una mayor estabilidad térmica.

\begin{tabular}{|c|c|c|c|c|c|}
\hline & $\mathrm{K}$ & Sup. & K*Sup & $K^{*}$ Sup ${ }^{-A T}$ & \\
\hline Componente & & & & $\mathrm{Dia}$ & Noche \\
\hline Muro Pq & 1,62 & 11,96 & 19,34 & 19,34 & 19,34 \\
\hline Muro P2 & 0,00 & 9,10 & 0,00 & 0,00 & 0,00 \\
\hline Muro P3 & 1.60 & 11,96 & 19,16 & 208,86 & $-114,97$ \\
\hline Muro P4 & 1,60 & 9,10 & 14,58 & 158,92 & $-87,48$ \\
\hline Muros Ext, Te & 4,82 & 42,12 & 53,08 & 387,12 & $-183,10$ \\
\hline Total Piso. & 0,00 & 14.91 & 0,00 & 0,00 & 0,00 \\
\hline Compuerta ho & 0.47 & 0.11 & 0,05 & 0,57 & $-0,31$ \\
\hline Compuerta ve & 0.47 & 0.11 & 0.05 & 0.57 & $-0,31$ \\
\hline Total Comp & 0.47 & 0,22 & 0.10 & 1,14 & 1,14 \\
\hline Total Techo & 0.00 & 14.91 & 0,00 & 0,00 & 0,00 \\
\hline TOTAL & 4,50 & 72,16 & 94,33 & 388,26 & $-181,97$ \\
\hline
\end{tabular}

\begin{tabular}{|c|c|c|c|c|c|}
\hline \multicolumn{6}{|l|}{ Ladrillo-Caso 6} \\
\hline Componente & & & & Dig & Noche \\
\hline Muro P1 & 1,68 & 14,56 & 24,47 & 266,70 & $-146,81$ \\
\hline Muro P2 & 1,68 & 13,78 & 23,16 & 23,16 & 23,16 \\
\hline Muro P3/Cistt & 0,00 & 13,78 & 0,00 & 0,00 & 0,00 \\
\hline Muro P4/Cons & 1,05 & 14,56 & 15,36 & 167,41 & $-92,15$ \\
\hline Muros Ext. Td & 4,42 & 56,68 & 62,98 & 457,26 & $-215,80$ \\
\hline Total Piso & 0,00 & 29,68 & 0,00 & 0,00 & 0,00 \\
\hline Compuertas & 0,46 & 0,11 & 0,05 & 0,55 & $-0,30$ \\
\hline Total Techo & 0,00 & 29,68 & 0,00 & 0,00 & $0, \infty$ \\
\hline TOTAL & 4,88 & 116.15 & 63,03 & 457,81 & $-216,10$ \\
\hline
\end{tabular}

Tabla 2 : Componentes construct|wos de la enwolvante del caso 3. Area transmitancias respectivas.

Tabla 3: Componentes constructiwos de a envolvente del caso 6. Area y transmitancias respectivas

b) El porcentaje de superficie de la EV en contacto directo con el exterior (\%)

En ambos casos los elementos de la envolvente en contacto con el entorno exterior son los muros. Los elementos horizontales se comparten con otras unidades de conservación, por lo tanto el intercambio térmico entre éstas es igual a cero. En el caso de adobe, el $45,76 \%$ de su envolvente intercambia energía térmica con el ambiente exterior. El porcentaje en el caso de ladrillo es algo menor, igual a $36,93 \%$. La envolvente del caso de adobe, está un $9 \%$ más expuesta que la del segundo caso, de ladrillo.

Las mayores cargas diurnas y nocturnas se originan en el intercambio térmico por convección, en ambas envol- 
ventes. En horas de la noche particularmente, la temperatura superficial desciende y el gradiente térmico acelera la disipación de calor hacia el entorno más fresco. Se observa que la envolvente de ladrillo tiene mayor cantidad de intercambio térmico al entorno, a pesar de disponer menor superficie exterior que el caso de adobe.

c) El volumen almacenado y variación térmica $(\Delta T)$

La carga térmica total transferida por la envolvente, se distribuye en el volumen de vino almacenado. La capacidad térmica del vino es semejante a la del agua, por lo cual se requieren $1 \mathrm{cal}$ para elevar $1^{\circ} \mathrm{C}$ su temperatura. La variación térmica horaria indica que la temperatura del vino en ambas envolventes oscilan $+0,02 \mathrm{~K}^{*} \mathrm{~h}$. En horas de la noche la variación térmica horaria del vino en ambas vasijas es semejante, con -0,20 para el caso de adobe y -0,22 para el caso de ladrillo. La diferencia de volumen entre ambos casos es del doble, con $24000 \mathrm{~L}$ (EV adobe) y $50000 \mathrm{~L}$ (EV ladrillo), cada uno.

d) Relación entre el volumen almacenado y la superficie de envolvente.

Esta relación, conocida como factor de forma (FF) es aproximada en ambos casos. Para EV adobe, este valor alcanza 2,32 m; mientras, para EV ladrillo el mismo es algo superior, igual a $2,89 \mathrm{~m}$. Sin embargo esta diferencia puede ser despreciada y se podría decir que los casos analizados son proporcionales, a escalas distintas.

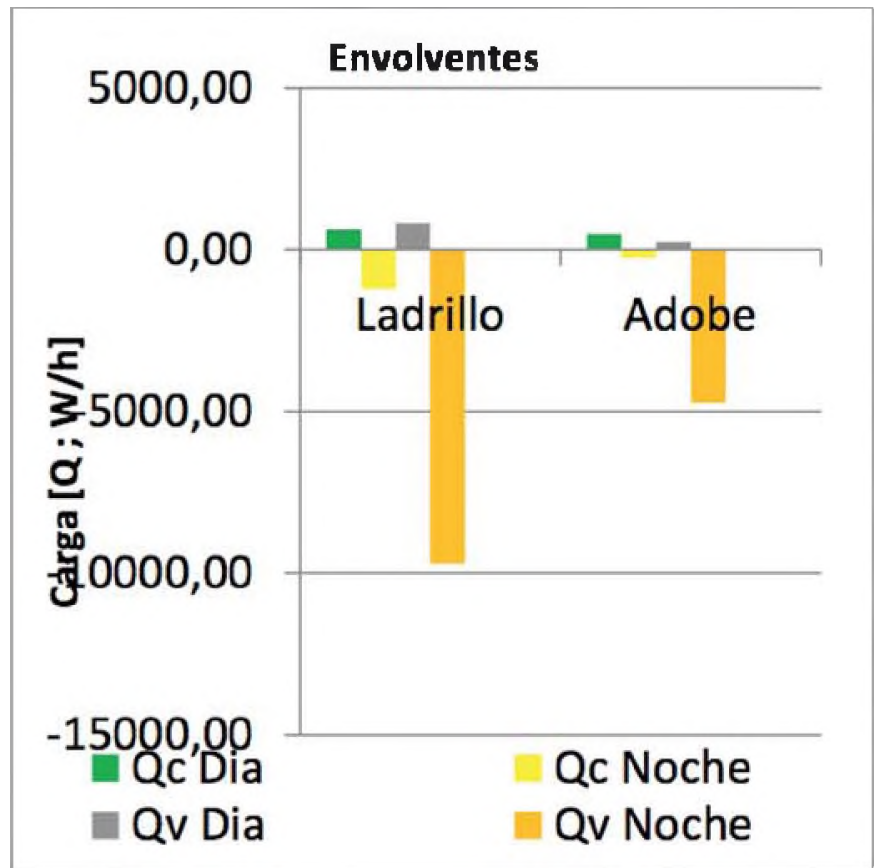

Figura 4: Cargas termicas para cada enwolvente, por modo y momento del ciclo de 24 horas.

\begin{tabular}{|l|l|r|r|}
\hline \multicolumn{4}{|c|}{ INTERCAMBIO TERMICO ENTORNO [W] } \\
\hline Modo & EV & \multicolumn{1}{l|}{ Ladrillo } & Adobe \\
\hline CONDUCCIONN & Qc Dia & 623,28 & \multicolumn{1}{|c|}{501,07} \\
\hline & Qc Noche & $-1208,88$ & $-244,07$ \\
\hline CONVECCIÓN & Qv Dia & 812,71 & 225,73 \\
\hline & Qv Noche & $-9703,92$ & $-4738,04$ \\
\hline EVAPORACIÓN & Qevap Dia & 0,00 & 0,00 \\
\hline & Qevap Noche & & - \\
\hline Q (t,v)EDILICIA & Dia & 1408,17 & 677,89 \\
\hline & Noche & $-10821,94$ & $-4891,26$ \\
\hline QEDILICIA+QR=E - Qame & Dia & 1048,39 & 514,13 \\
\hline & Noche & & 0,00 \\
\hline AUTOREGULACION [\%] & QA(E)Dia & 1,98 & 31,37 \\
\hline & QR(E)Noche & 768,51 & 721,54 \\
\hline$\Delta T^{*} h$ [K] & Dia & 0,02 & 0,02 \\
\hline & Noche & $-0,22$ & $-0,20$ \\
\hline
\end{tabular}

Tabla 4: Energia térróca intercambiada por la envolvente de los casos analiza dos, por meco de transíaremeia de calor.

\section{Conclusiones:}

Considerando las variables analizadas, desde el punto de vista termo-energético no existen diferencias considerables entre ambos tipos de envolventes. Técnicamente, es igualmente apropiada a la conservación del vino una envolvente de adobe asi como una de ladrillo. Esta conclusión no desestima que existe una diferencia en el desempeño térmico de ambos casos. En el seguimiento térmico del caso o se observa una respuesta más veloz en la variación térmica del vino a un estímulo externo. Este comportamiento se verifica en el modelo térmico al resultar una amortiguación térmica superior en el caso 3. La diferencia en el retardo térmico $[\varphi]$ entre materiales señala al adobe como el más estable, con seis horas de diferencia con el ladrillo. Sin embargo en un período extenso, el vino se estabiliza a la temperatura media del aire, en ambos casos.

Las diferencias económicas son reducidas desde la perspectiva de la inversión, dado que el costo del millar de ladrillos podría equipararse a la elaboración tradicional de un bloque de adobe. La variable económica en términos de rendimiento relacionando el costo del m2 de construcción con el volumen que almacena indica que el rendimiento económico es un $22,6 \% / \mathrm{L}$ mayor para EV de ladrillo, no por su materialidad sino por el FF, con el cual es posible almacenar mayor volumen de vino que en el caso 3. La proporción de la forma en términos energéticos es determinante. En envolventes de

conservación es favorable que los muros de mayor superficie asi como las losas y cubiertas sean compartidos con otras unidades de almacenamiento. Esto favorece a una disminución de los costos económicos de construcción y optimiza la performance térmica de la envolvente sin introducir gastos en aislación térmica, 
aprovechando el efecto amortiguador de las masas de vino incrementadas en la aglomeración de unidades de conservación del vino.

\section{Referencias:}

-Blasco Lucas I., Hoesé L., Pontoriero D. (2007). Procedimiento "PROMEDI-HTL-V3" para análisis comparativos de mediciones higrotérmicas y lumínicas. Avances en Energias Renovables y Medio Ambiente, Vol. 11., pp. 08.01-08.06.

-Colombié et al (2006) Modeling of Heat Transfer in Tanks during wine-making fermentation. Food Control (18), 2007, págs. 953-960 -INSTITUTO ARGENTINO DE NORMALIZACIÓN (1996) IRAM11603 ClasificaciónBioambiental de la República Argentina. Buenos Aires, Argentina.

-Instituto de Desarrollo Rural (IDR, 1999) Caracterización de la Cadena Agroalimentaria de Vitivinicultura de la Provincia de Mendoza. Instituto Nacional de Tecnolo gía Agropecuaria (INTA) Texto completo en http://www.inta.gov.ar

-Instituto Nacional de Vitivinicultura San Juan (INV, 2008) Listado de bodegas elaboradoras de San Juan.

Ministerio de la Producción de la Provincia de San Juan. Calidad San Juan (2010)

-Informe Final de la Planificación Estratégica de la cadena Productiva de Vinos Básicos en Sam Juan -Ministerio de Relaciones Exteriores y de Culto de la República Argentina (2012) Informe Sectorial. Sector Vitivinicola.

-Oreglia, F. (1978) Enología Teórico-práctica. Ediciones Instituto Seliciano de Artes Gráficas, Buenos Aires. Págs. 185-204. 7

-Ramos Sanz, A. (2013) Caracterización de la demanda energética en bodegas. Propuesta de indicadores de eficiencia energética. Actas de la XXXVI Reunión de Trabajo de la Asociación Argentina de Energías Renovables y Medio Ambiente. Vol. 1; pp. 07.09-07.16, 2013. República Argentina. ISBN 978-987-29873-0-5

-Ramos Sanz, A. (2014) El Confort del Vino en la Envolvente Industrial. Estudio de casos de vasijas vinarias de Bodegas argentinas. Editorial Publicia, Saarbrücken, Alemania, 2014. ISBN 978-3-639-55635-3.N

-Ramos Sanz, A. y Blasco Lucas, I. (2011) Condiciones Higrotérmicas de fermentación de vinos comunes y finos de variedades blancas y tintas. Revista AVERMA ISSN 0329-5184.N

-Ramos Sanz, A. y Kuchen, E. (2013) La incidencia del clima árido en dos instancias de vinificación. Actas de la XXXVI Reunión de ASADES Tucumán, 2013ISBN 978-987- 29873-0-5 y Revista AVERMA ISSN 0329-5184.N

-Udaquiola, S; et al (2006) Mass energy balances in the wine industry. 2nd Mercosur Congress on Chemical Engineering. 